\title{
A Probabilistic Approach to Model-Based Adaptive Control for Damping of Interarea Oscillations
}

\author{
Rajat Majumder, Student Member, IEEE, Balarko Chaudhuri, Student Member, IEEE, and \\ Bikash C. Pal, Senior Member, IEEE
}

\begin{abstract}
This paper demonstrates an adaptive control strategy for damping interarea oscillations in a large power system model, employing a probabilistic approach to model-based control. The scheme accounts for the uncertain nature of the post-disturbance dynamics of the system for computing the control moves. A number of linearized plant models are considered to represent the system dynamics following the probable contingencies. Conventional observer-based state feedback controllers are designed to achieve the desired performance for each of these models. This strategy has been used to design and test a damping controller for a thyristor controlled series compensator (TCSC) device installed in a 16-machine, 68-bus system model. The control scheme worked satisfactorily following possible disturbances without any prior knowledge about the specific post-disturbance dynamics.
\end{abstract}

Index Terms-Adaptive, Bayesian approach, interarea oscillations, robustness, thyristor controlled series compensator (TCSC).

\section{INTRODUCTION}

$\mathbf{L}$ OW-frequency interarea oscillations $(0.2-1.0 \mathrm{~Hz})$ are common in large interconnected power systems, and they have been of concern for many years [1]. Stabilization of the oscillatory modes via excitation systems has been extensively investigated [2]. The most cost-effective solution to the problem is the application of power system stabilizers (PSSs) in generator excitation control systems. Proper tuning and coordination of multiple PSSs can adequately damp out oscillations that involve both local and interarea modes.

A thyristor controlled series capacitor (TCSC) or a static var compensator (SVC) has long been used for effective power flow control and dynamic voltage support of the system. These devices belong to a family of controllable devices known as flexible ac transmission systems (FACTS) devices. The FACTS technology is receiving growing importance as an alternative to transmission system reinforcement, which is restricted due to economic and environmental reasons [3]. Besides line power flow and system voltage control, supplementary control is being added to these FACTS devices to damp out the interarea oscillations at not much additional cost.

Over the years, the main challenge for power system damping control designers has been to ensure satisfactory performance over a wide range of operating conditions. The famous blackout on 10 August 1996 in the Western System Coordinating Council

Manuscript received May 25, 2004. This work was supported by EPSRC, Swindon, U.K., under Grant GR/S06158. Paper no. TPWRS-00572-2003.

The authors are with the Department of Electrical and Electronic Engineering, Imperial College London, London, U.K. SW7 2BT (e-mail: r.majumder@imperial.ac.uk; b.chaudhuri@imperial.ac.uk; b.pal@imperial.ac.uk).

Digital Object Identifier 10.1109/TPWRS.2004.840408
(WSCC) system was a consequence of undamped power oscillations [1]. This has given the network operators and researchers a wake-up call to come up with a comprehensive solution to this damping control design problem. The basic question is as follows: Can the control strategy ascertain damped response of the system for a range of probable contingencies? The answer is not always yes, as the conventional damping control design approach considers only the nominal operating condition of the system [2] during the controller synthesis stage. Classical techniques such as Proportional and Integral (PI) and the root-locus approach ensure desired damping performance for a particular operating condition. The controllers obtained from these approaches are simple but, at times, might fail to produce adequate damping for other operating conditions, if not properly tuned.

Researchers, over the years, have proposed several adaptive control structures for power system stabilizers to address this issue. Malik et al. [4] applied the model reference adaptive control (MRAC) strategy where the error between the power system response and the reference model output is used to modify the controller parameters such that the plant behavior is driven to match the behavior of the reference model. A self-tuning control (STC) of PSS has been reported by Pahalawaththa et al. [5], where the amount of pole shifting is adjusted depending upon the system conditions. Bandyopadhayay et al. [6] have presented a gain scheduling control (GSC) scheme for PSS, where the controller parameters are tuned based on the minimization of the distance between the current and the desired operating points.

An alternative control design methodology using $\mathcal{H}_{\infty}$ technique has been proposed [7], [8] to tackle the robustness issue. It does guarantee robustness at least for a range of operating conditions. However, if the uncertainties are not modeled properly in a structured manner, the controllers tend to be too conservative and might ask for unacceptably large control effort under certain situations.

Model predictive control offers an effective solution to this problem by ensuring optimal control effort under varying operating conditions, provided the initial values of the system states are specifically known. In power systems, it is almost impossible even to estimate the initial values of the states following a large disturbance. This is because even if the models corresponding to the predisturbance and post-disturbance behavior is known, it is very difficult to model the behavior during the disturbance (e.g., fault). What one can hope for is to devise a mechanism that combines several known post-disturbance models to produce a model that can closely match the system dynamics for an arbitrary contingency. This argument can be extended for controllers 


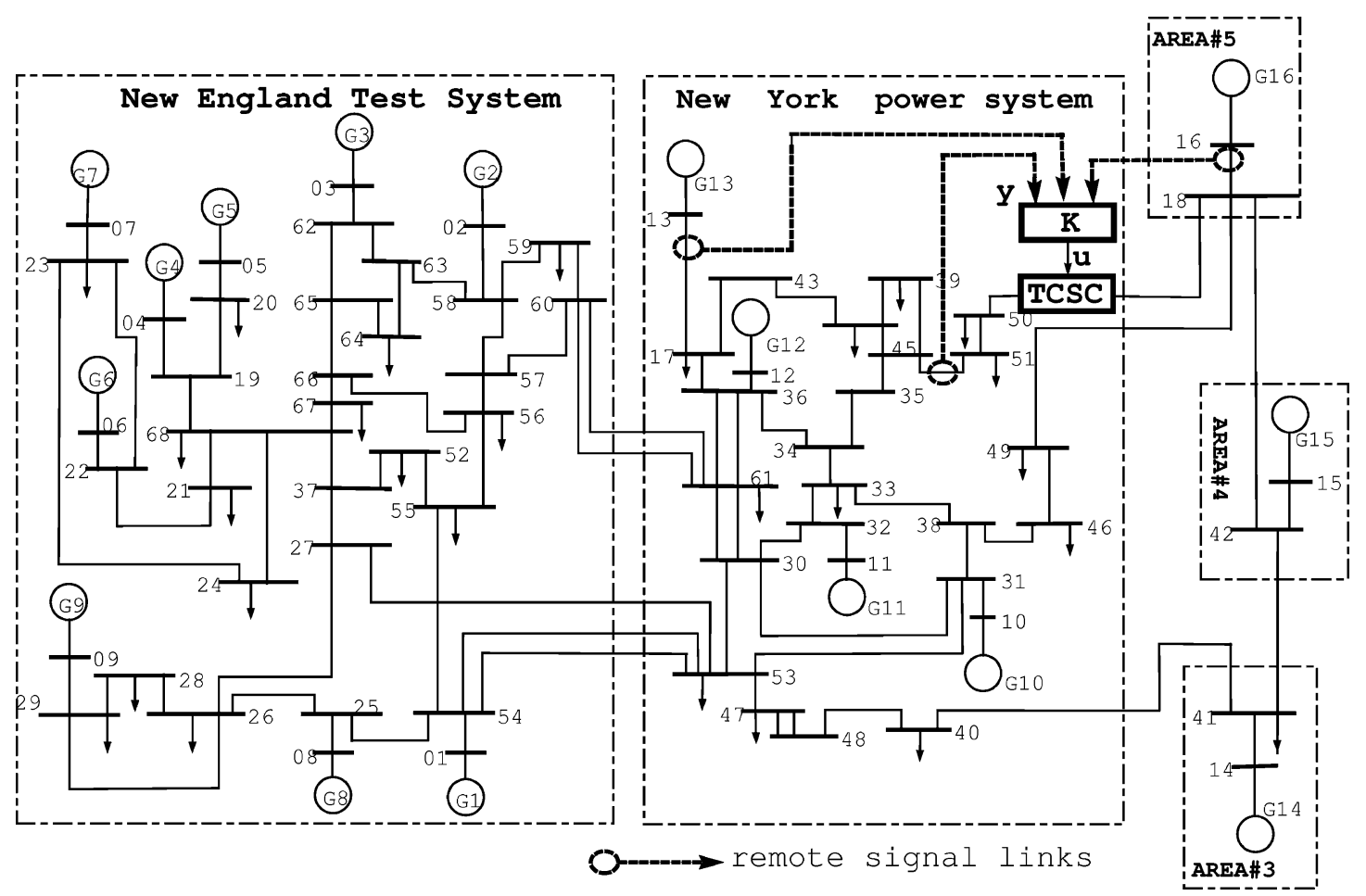

Fig. 1. Sixteen-machine, 5-area study system with TCSC.

also. That means that one needs to have a set of predesigned controllers for different post-disturbance system models. These sets are known as model bank and controller bank. It is not difficult to have an idea about the model bank from past statistics and experience of the system operators. The larger the number of elements in the model bank, the greater the accuracy in predicting the unknown dynamics. This might be difficult to implement on line because of large computational efforts. On the other hand, intuitively, it can be said that in any actual system behavior, the influence of all the elements in the model and controller bank will not be uniform. The Bayesian probabilistic approach is a mathematical abstraction of the intuition, which enables quick detection of the trend in the post-contingency dynamic behavior by providing a weight vector for blending the control action the pretuned controllers. This control scheme is designed and tested for a TCSC. The TCSC is installed in a 16-machine, 68-bus power system model, as described in the next section.

\section{STUDY SYSTEM}

A 16-machine, five-area study system, shown in Fig. 1, is considered for the damping control design. This is a reduced-order model of the New England and New York interconnected power systems of the 1970s. In the current electricity business situation, many regional transmission operators (RTOs), independent system operators (ISOs), and regional generation companies have been formed. However, the dynamic behavior of the system would not change appreciably, as they still operate in an interconnected fashion.

The first nine machines (G1-G9) in Fig. 1 are a simple representation of the New England Test System (NETS) generation. Machines G10-G13 represent the New York Power System (NYPS) generation. The last three machines (G14-G16) are the dynamic equivalents of the three neighboring areas connected to the NYPS. All the machines are modeled with four windings on the rotor, i.e., field circuit, one damper in the d-axis, and two dampers in the q-axis. The first eight machines have slow excitation (IEEE type DC1A), whilst machine G9 is equipped with a fast-acting static excitation system (IEEE ST1A) [2]. This particular machine is also assumed to have a speed-input power system stabilizer, described in [9]. The loads are all assumed to be of constant impedance (CI) type for the base case. The data for bus, line, and machine exciters and loads are taken from [9] and [10]. NYPS is required to import $1550 \mathrm{MW}$ from Area 5. To facilitate this power transfer, a TCSC is installed in the line between buses 18 and 50 near bus 50 . To realize the desired power flow scenario, the percentage compensation of the TCSC is set to 50. The interarea modes are obtained from the eigen-value analysis and are displayed in Table I.

There are four interarea modes, the first three of which are critical. Mode 4, on its own, settles in less than $10 \mathrm{~s}$, as its frequency is comparatively higher than the other modes (the higher the oscillation frequency, the faster the settling for a given damping ratio). Since the influence of mode 4 does not last beyond $10 \mathrm{~s}$ and an overall system settling time of 10-12 s is perfectly acceptable, it is not required to provide additional damping to this mode. It was decided to provide damping control action to the three critical interarea modes through the TCSC.

A modal observability and residue analysis [11] was carried out to identify the most effective stabilizing signals for the TCSC. The residue is the product of modal observability and modal controllability. The higher the residue, the lower the control effort required. The modal controllability is fixed for a fixed location of the TCSC. The modal observability depends 
TABLE I

INTERAREA MODES OF THE STUDY SYSTEM

\begin{tabular}{c||c}
\hline$\zeta$ & $f(\mathrm{~Hz})$ \\
\hline \hline 0.0626 & 0.3913 \\
\hline 0.0435 & 0.5080 \\
\hline 0.0554 & 0.6232 \\
\hline 0.0499 & 0.7920 \\
\hline
\end{tabular}

on the feedback signal used. Therefore, the most effective signals from remote locations were searched for using the modal observability analysis. Table II shows the normalized residues for the three modes with power flow signals available from different lines.

It is evident from the table that although mode 1 is noticeably observable in the locally available signals $P_{50,51}, P_{50,18}$ (shown in boldface), the observability of the other two modes are poor. Here, $P_{50,51}$ and $P_{50,18}$ indicate the power flow in the lines between buses 50-51 and buses 50-18, respectively. The results, displayed in Table II, indicate that $P_{51,45}, P_{18,16}$, and $P_{13,17}$ (shown in boldface) are the most effective signals for mode 1, mode 2 , and mode 3 , respectively, where $P_{51,45}, P_{18,16}$, and $P_{13,17}$ indicate the power flow in the lines between buses 51-45, 18-16, and 13-17, respectively.

\section{Control Design Based on Model-Based PROBABILISTIC APPROACH}

This approach was originally introduced by Lainiotis [12]. Subsequently, it has been used for the control of aircraft [13] and for regulation of hemodynamic variables [14], [15]. The basic motivation behind applying this scheme in a power system is that it can achieve the desired performance without any prior requirement to identify the post-disturbance dynamics before initiating the control action. The assumption, though, is that the actual system response can be represented by a single or a suitable combination of a finite number of linearized models. For each model, an observer-based state feedback controller is designed a priori to meet the specified performance objective. Using a Bayesian approach, the current probability of each model representing the actual system response is calculated, and the results are used to determine the subsequent control moves. The probabilities are iteratively computed at every instant by improving upon the probability computed from the previous instant [16]. The control move of an individual controller is assigned a weight based on the probability of that particular model, representing the actual response. Thus, at each instant, the resulting control action is the probability-weighted average of the control moves of the controllers.

\section{A. Model Bank}

The major contingencies in a power system could be the outage of key tie lines, sudden changes in the power flow through tie lines, or changes in load characteristics. It is always difficult to predict the possible contingencies. However, a probable list can be anticipated from previous experience in system operation. Each of the post-contingency behaviors of the system can be included in the model bank in the form of a
TABLE II

NORMALIZED RESIDUES FOR REAL POWER FLOW SIGNALS FROM DIFFERENT LINES

\begin{tabular}{c|c||c|c||c|c}
\hline \multicolumn{2}{c||}{ Mode 1 } & \multicolumn{2}{c||}{ Mode 2 } & \multicolumn{2}{c}{ Mode 3 } \\
\hline Line & Residue & Line & Residue & Line & Residue \\
\hline \hline $\mathbf{5 1}-\mathbf{4 5}$ & 1.00 & $\mathbf{1 8}-\mathbf{1 6}$ & 1.00 & $\mathbf{1 3}-\mathbf{1 7}$ & 1.00 \\
\hline $\mathbf{5 0}-\mathbf{5 1}$ & 0.67 & $41-14$ & 0.79 & $36-17$ & 0.67 \\
\hline $\mathbf{5 0}-\mathbf{1 8}$ & 0.63 & $42-18$ & 0.75 & $60-61$ & 0.34 \\
\hline $\mathbf{3 5}-\mathbf{3 4}$ & 0.63 & $41-42$ & 0.55 & $53-30$ & 0.30 \\
\hline $45-35$ & 0.63 & $53-30$ & 0.34 & $61-36$ & 0.29 \\
\hline $\mathbf{3 4}-36$ & 0.57 & $53-47$ & 0.31 & $54-53$ & 0.29 \\
\hline $\mathbf{5 3}-47$ & 0.56 & $51-45$ & 0.29 & $\mathbf{5 0}-\mathbf{5 1}$ & 0.27 \\
\hline $\mathbf{3 6}-34$ & 0.55 & $\mathbf{5 0}-\mathbf{5 1}$ & 0.29 & $\mathbf{5 0}-\mathbf{1 8}$ & 0.25 \\
\hline $\mathbf{5 3}-\mathbf{5 4}$ & 0.50 & $\mathbf{3 6}-17$ & 0.28 & $34-36$ & 0.24 \\
\hline $41-40$ & 0.49 & $\mathbf{5 0}-\mathbf{1 8}$ & 0.28 & $68-37$ & 0.21 \\
\hline
\end{tabular}

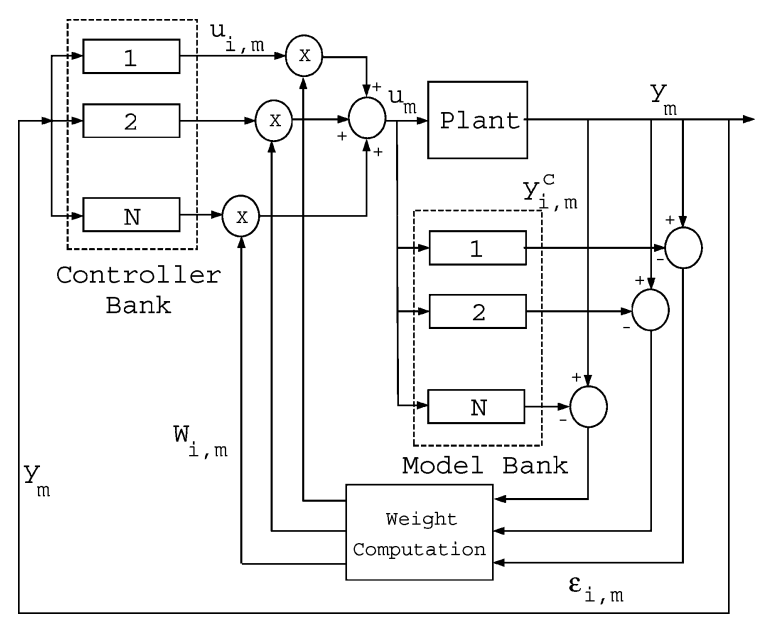

Fig. 2. Schematic diagram of the control algorithm.

linear model (see Fig. 2). The more linearized models included in the model bank, the more accurate the identification of actual system dynamics following any arbitrary contingency. From the viewpoint of practical implementation, as the number of models increases, the computational burden will also increase. So, the number of elements in the model bank has to be limited. In this research, while building the linearized model of the system under different operating conditions, a few credible contingencies are considered. For example, a fault in the two main transmission corridors between buses 53-54 and 60-61 has severe contingencies. Also, the sudden change in power flow through a line has been included. In this way, nine probable system models have been considered. The operating scenarios and corresponding model identifiers are summarized in Table III.

Model 1 is for the nominal operating condition with a 700-MW power transfer through the tie line and all the tie lines in place. Models 2-4 reflect the situation with one of the tie lines between buses 53-54, 60-61, and 27-53 switched off, respectively. Models 5 and 6 correspond to 100 and $900 \mathrm{MW}$ through tie line 60-61, respectively. Models 7 and 8 are a combination of constant current (CC), constant power (CP), and constant impedance (CI) load. In model 9, dynamic induction motor type load is considered at bus 41 . The model identifiers described in Table III have been used to refer to the specific models throughout this paper. 
TABLE III

MODEL BANK

\begin{tabular}{c||c||c||c}
\hline Model & Tie-Line flow (MW) & Outage of line & Type of load \\
\hline \hline 1 & 700 & No outage & CI \\
\hline 2 & 700 & $53-54$ & CI \\
\hline 3 & 700 & $60-61$ & CI \\
\hline 4 & 700 & $27-53$ & CI \\
\hline 5 & 100 & No outage & CI \\
\hline 6 & 900 & No outage & CI \\
\hline 7 & 700 & No outage & CI + CC \\
\hline 8 & 700 & No outage & CI + CP \\
\hline 9 & 700 & No outage & Dynamic load \\
\hline
\end{tabular}

\section{B. Control Algorithm}

The recursive algorithm uses a bank of linearized plant models to capture the possible system dynamics following a disturbance. An observer-based state feedback controller $k$ is designed for each plant model $k$. All of these controllers are put in a controller bank, shown in Fig. 2. At each simulation step, the actual plant response is compared with the response of the linearized models, which are driven by the same control input. The difference in the response of each model with respect to the actual system response is used to generate individual model mismatch residuals. Using these residuals, the probability of each model representing the actual system response is computed. Based on the probabilities, suitable weights are assigned to individual control moves such that the less probable models carry less weight. This ensures that the controllers designed for less probable models influence the final control move to a lesser extent. The resultant control action is, thus, a probability-weighted average of the control moves of each individual controller. At each stage of the recursive algorithm, primarily, two tasks are performed, i.e., calculation of probability using a Bayesian approach and assigning suitable weights based on the value of the probability.

1) Calculation of the Probability: Bayesian Approach: The recursive Bayes theorem [17] is used for computing the probability of each model in the bank. The theorem calculates the conditional probability of the $i$ th model in the model bank being the true model of the plant, given this population of model. A number of power system outage studies [18] have suggested that power system disturbance behavior can be modeled via the Gaussian approach. Therefore, the probabilities are assumed to be stochastic and Gaussian in nature and, thus, take a form of the exponential of the negative square of the residuals [16]. At the $m$ th step, the probability for the $i$ th model is calculated as follows:

$$
p_{i, m}=\frac{\exp \left(-\frac{1}{2} \varepsilon_{i, m}^{T} C_{f} \varepsilon_{i, m}\right) p_{i, m-1}}{\sum_{j=1}^{N} \exp \left(-\frac{1}{2} \varepsilon_{j, m}^{T} C_{f} \varepsilon_{j, m}\right) p_{j, m-1}}
$$

where

$$
\varepsilon_{i, m}=y_{m}-y_{i, m}^{c}
$$

is the error or model residual at the current step. $y_{m}$ denotes the vector of measured outputs of the plant, which corresponds to measured signals $P_{51,45}, P_{18,16}$, and $P_{13,17}$ described in Section II. $N$ is the total number of models in the model bank, and
$C_{f}$ is the convergence factor that is used to tune the rate of convergence of the probabilities.

Large values of $C_{f}$ will magnify the model residuals and cause an acceleration of convergence to a single model. The recursion is initialized by assigning equal probability $(1 / \mathrm{N})$ to all the models in the bank. At each iteration, new probabilities are calculated, improving upon the probability computed at the previous iteration. One major advantage with this algorithm is that it is computationally inexpensive. An additional benefit is that the poor models are rejected exponentially, thereby allowing a widely varying set of models without necessarily leading to a large drop in controller performance, even during the initial stages [19]. To summarize, for a given set of models, the above algorithm recursively determines the probability that the $i$ th model is the true plant model. The computation is based on the present model residuals with respect to the actual system response and the previous probabilities for each model [16].

2) Calculation of Weights: Based on the probability of individual models, calculated during each recursive step, suitable weights are assigned to the control moves of each of the controllers. The model with a higher probability is assigned a higher weight and vice versa. One of the features of this Bayesian approach is that it can only assume a steady-state probability of either zero or one, and consequently, the algorithm converges to a single model. However, due to the uncertainties associated with a practical power system, it is unlikely that any single model in the model bank would be exactly equivalent to the system under control, and hence, proper blending of control action is often required. Models attaining a probability of zero cannot enter the subsequent recursions and, hence, an artificial cutoff $\beta_{\min }$ is used to keep them alive. At the $m$ th step, the $i$ th model is assigned a weight $W_{i, m}$ such that

$$
W_{i, m}= \begin{cases}\frac{p_{i, m}}{\sum_{j=1}^{N} p_{j, m}}, & \forall p_{i, m}>\beta_{\min } \\ 0, & \forall p_{i, m}<\beta_{\min } .\end{cases}
$$

For models with $p_{i, m}<\beta_{\min }$, the probability is reset to $p_{i, m}=\beta_{\min }$, and these models are then excluded from being weighted. At the $m$ th iteration, the resulting probability-weighted control move is computed as

$$
u_{m}=\sum_{j=1}^{N} W_{j, m} \cdot u_{j, m} .
$$

$u_{m}$ is the supplementary control input to the TCSC, which corresponds to $u$ in Fig. 1.

\section{Design of Controller Bank}

The first step toward the implementation of the control scheme is to design and tune one separate controller for each of the nine linearized system models, described in Section III. The order for each of these plant models was 132. To facilitate control design, the nominal plant was reduced to seventh-order equivalents without losing much of the relevant information in the frequency range of interest $(0.1-1.0 \mathrm{~Hz})$, as illustrated in Fig. 3. 


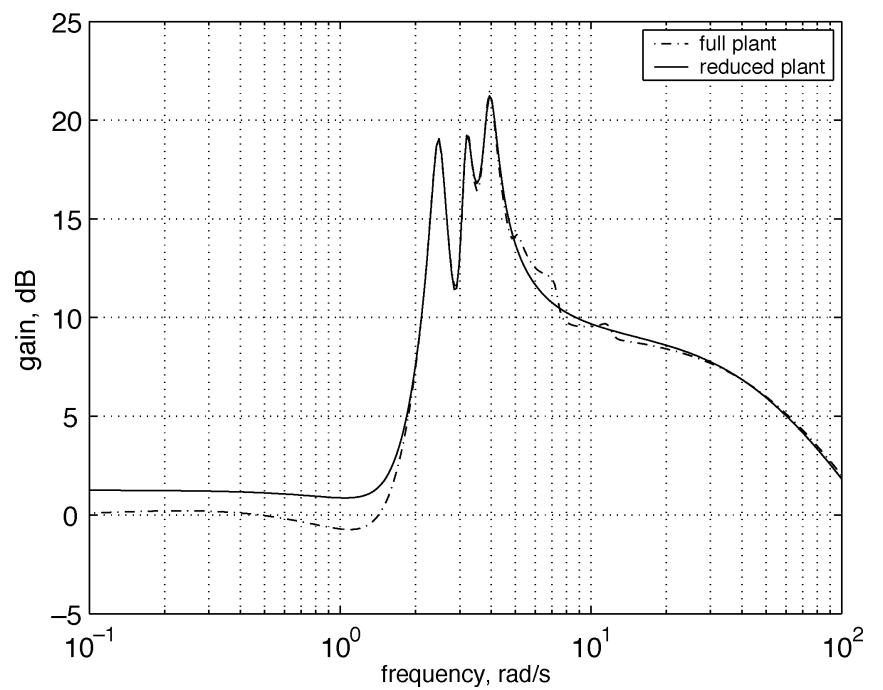

Fig. 3. Frequency response of the plant.

To improve the damping ratio of the critical interarea modes, an observer-based state feedback controller was designed for each of the models. The state feedback gain was determined to ensure settling of interarea oscillations within 10-12 s, a criterion followed by the power system utilities [1]. The "place" function available with the Control System Toolbox in Matlab was used to compute the gain. In power systems, all the states are not available for feedback, and hence, an observer was designed to derive the states from the measured outputs.

However, controllers designed using such a technique cannot maintain the desired performance level for a range of possible operating conditions. The performance of the designed controllers has been examined against different post-disturbance conditions described by the linearized models in the model bank. A few simulation results for plant model and controller combinations are shown in Figs. 4 and 5.

Model 1 represents the nominal operating condition or the base case as given in Table III. The closed-loop damping ratios for the base case with controller 1 is satisfactory as shown in Table IV. It can be seen from Fig. 4 that the model 1-controller 1 combination has a well-settled response, as expected. Similar satisfactory performances were obtained for models with the corresponding controller. As an example, the performance of model 3 is shown with controller 3 . However, the responses for model 2-controller 4, model 2-controller 9, model 3-controller 1, and model 3-controller 6, shown in Fig. 5, are inferior as oscillations of small magnitude continue beyond $15 \mathrm{~s}$. This has provided the motivation to adopt a probabilistic approach for improving the robustness under different operating conditions.

\section{Test Cases}

A conventional controller $k$, designed and tuned on the basis of model $k$, is not necessarily guaranteed to meet the desired performance specification for other models. Therefore, some mechanism needs to be devised for online identification of the unknown dominant dynamics following a disturbance and switch to an appropriately weighted combination of the controllers. Two situations can arise considering the uncertainty involved in a practical power system and the limit on the number
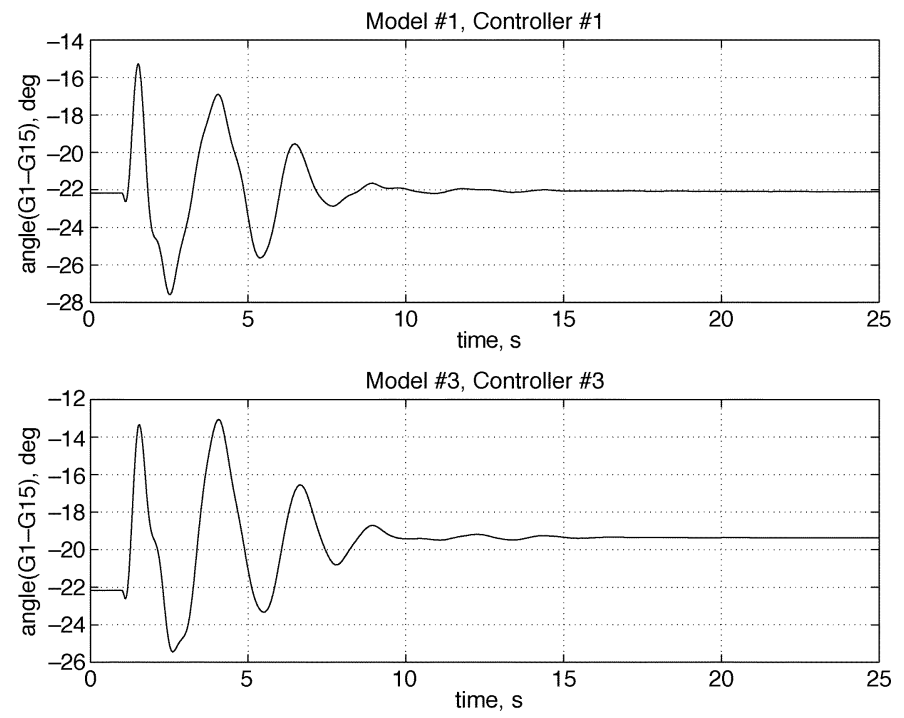

Fig. 4. Performance of conventional controllers.
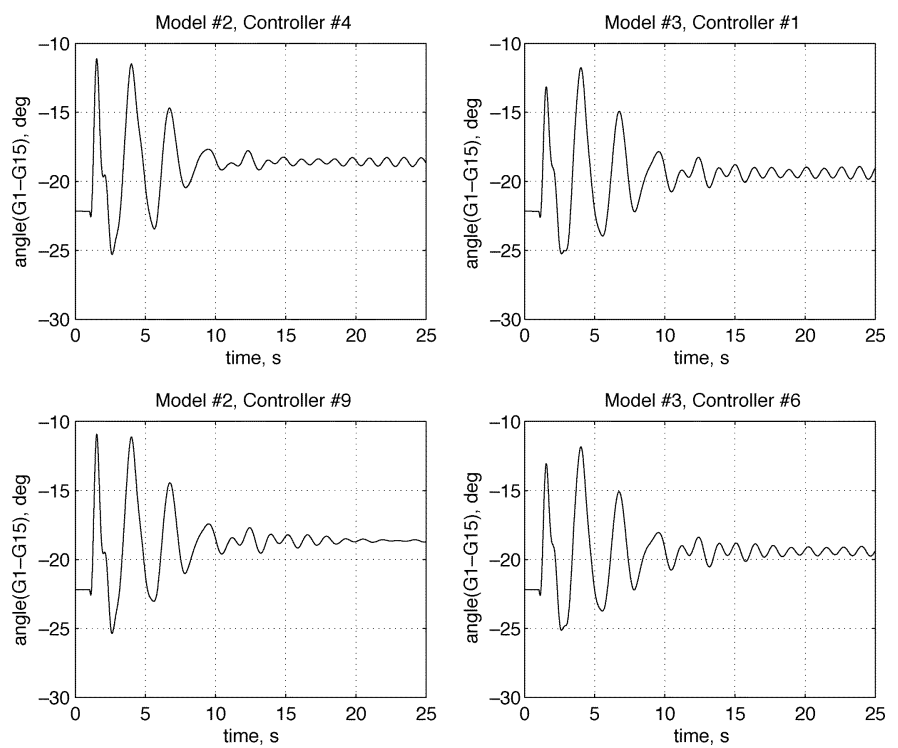

Fig. 5. Robustness test for conventional controllers.

TABLE IV

Close-Loop Damping Ratios in Base Case

\begin{tabular}{c||c}
\hline$\zeta$ & $f(\mathrm{~Hz})$ \\
\hline \hline 0.2079 & 0.3822 \\
\hline 0.1396 & 0.5189 \\
\hline 0.1346 & 0.6303 \\
\hline
\end{tabular}

of models that can be included in the model bank. In one case, the model corresponding to the dominant post-disturbance dynamics is likely to be present in the model bank, wherein the scheme should pick up the controller corresponding to that model with maximum weight. In the other case, the model representing the dominant post-disturbance dynamics is less likely to be present in the model bank, wherein the scheme should be able to ensure proper blending between the control moves of the existing controllers and achieve the desired performance criteria. These two test cases have been treated separately in this paper and are elaborated in the following subsections. 


\section{A. Test Case I}

For this test case, a three-phase line-to-ground fault was simulated at bus 53 for $80 \mathrm{~ms}$, followed by the opening of one of the tie lines connecting buses 53 and 27 (see Fig. 1). From Table III, it can be seen that the dynamics corresponding to this particular post-disturbance situation are captured by model 4 . All the nine models, including model 4, were kept in the model bank and the corresponding controllers in the controller bank. The objective was to see whether and how quickly the adopted probabilistic recursive algorithm could identify the dominant post-disturbance dynamics and apply the appropriate control action to achieve the desired performance.

\section{B. Test Case II}

For this test case, two simulation studies were done. In the first case, the same disturbance, as described in Test Case I, was considered. In the second case, a sudden partial loss in generation at generator G1 has been considered (see Fig. 1). Due to the uncertainty involved in a practical power system, it is unlikely that any single model in the model bank would be the exact equivalent of the system under control. Moreover, due to computational constraints, only a few out of the large number of possible models can be included in the model bank. To replicate either of these situations, model 4 and the corresponding controller 4 was deliberately removed from the banks for the first simulation study. The model corresponding to the second disturbance was not there in the model and controller bank in any way. The idea was to validate whether an appropriate blending of the remaining control moves is able to achieve the desired performance in the absence of the actual controller. This would demonstrate the ability of the control algorithm to pick up a proper blend of the relevant post-disturbance dynamics to closely mimic the actual system response.

\section{RESUlTS AND Discussions}

The simulations were performed in the Simulink environment of Matlab using a step size of $20 \mathrm{~ms}$ and a fourth-order Runge-Kutta solver. The results are separately presented for the two test cases.

\section{A. Case I}

The results of time domain simulation for Test Case I are shown in Figs. 6 and 7. The linearized model of the power system corresponding to the post-disturbance situation (model 4) was present in the model bank. As a result, the error residual for model 4 starts decreasing after few initial recursive steps, and consequently, the weight corresponding to this model goes up and attains a steady-state value of almost 1.0 (see Fig. 6). The aim, in this case, was to demonstrate the ability of the control scheme to identify the unknown dynamics and switch to the proper controller. This is why a relatively high magnitude of 0.005 is chosen for the convergence factor $C_{f}$ to quickly reject the unwanted models. Also, the artificial cut-off $\beta_{\min }$ is kept to a small value of 0.0001 to avoid retaining these unwanted models during subsequent recursive steps.

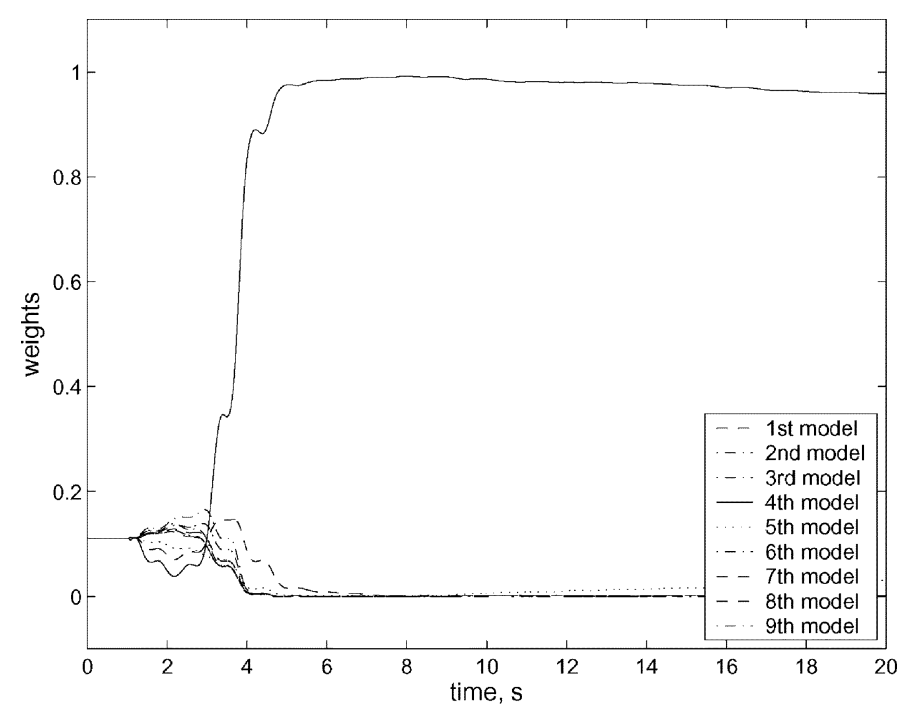

Fig. 6. Test case I: Variation of weights.
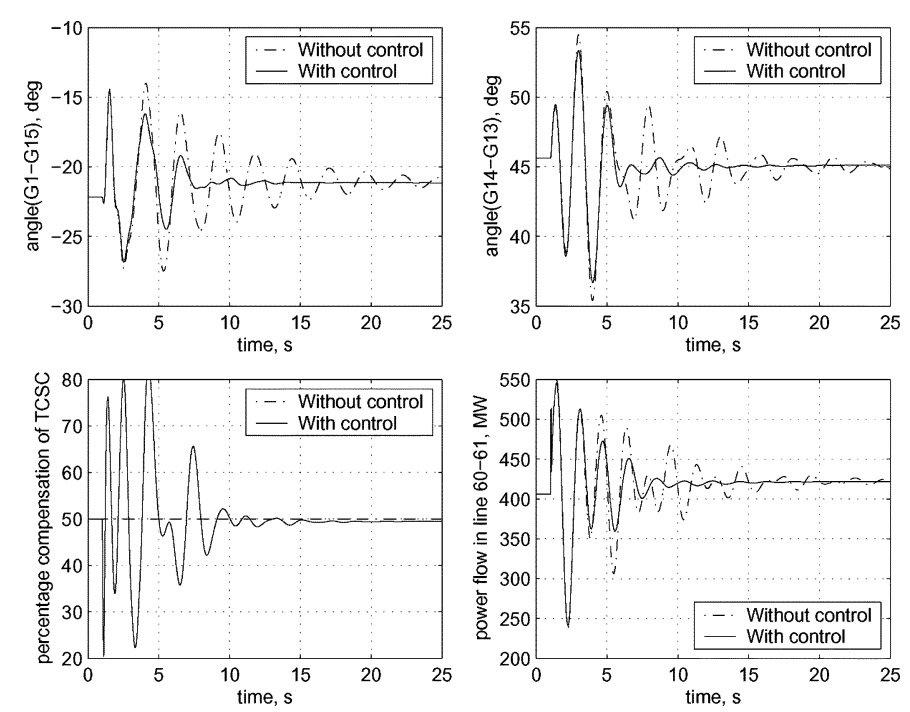

Fig. 7. Test case I: Dynamic response of the system.

The displays in Fig. 7 show the relative angular separation between machines G1-G15 and G14-G13. It can be seen that the lightly damped oscillations are settled in 12-15 $\mathrm{s}$ in the presence of the applied control scheme. Power flow between buses 60 and 61 also settles within the acceptable time frame. The resulting control is primarily dominated by controller 4 , owing to its higher weight. The simulation results illustrate that the control scheme is able to identify the dominant post-disturbance dynamics and switch the proper controller without any prior knowledge about the post-disturbance operating condition by using online recursive calculation of model probabilities and associated weights.

\section{B. Case II}

For these two simulation studies, the model governing the post-disturbance dynamics was not present in the model bank, and also, the corresponding controllers were also absent from the controller bank. 


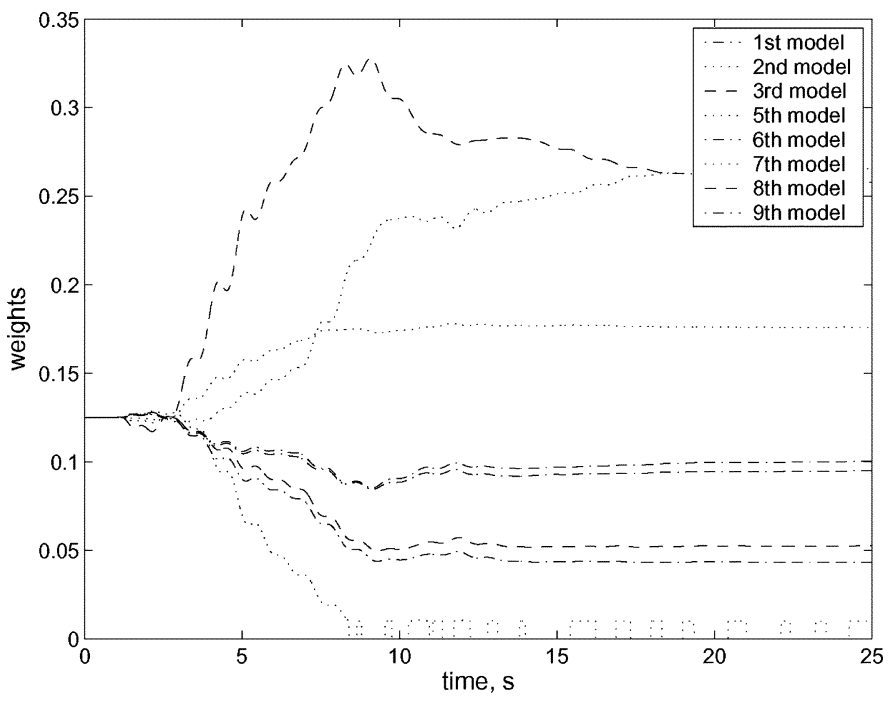

Fig. 8. Test case IIa: Variation of weights.
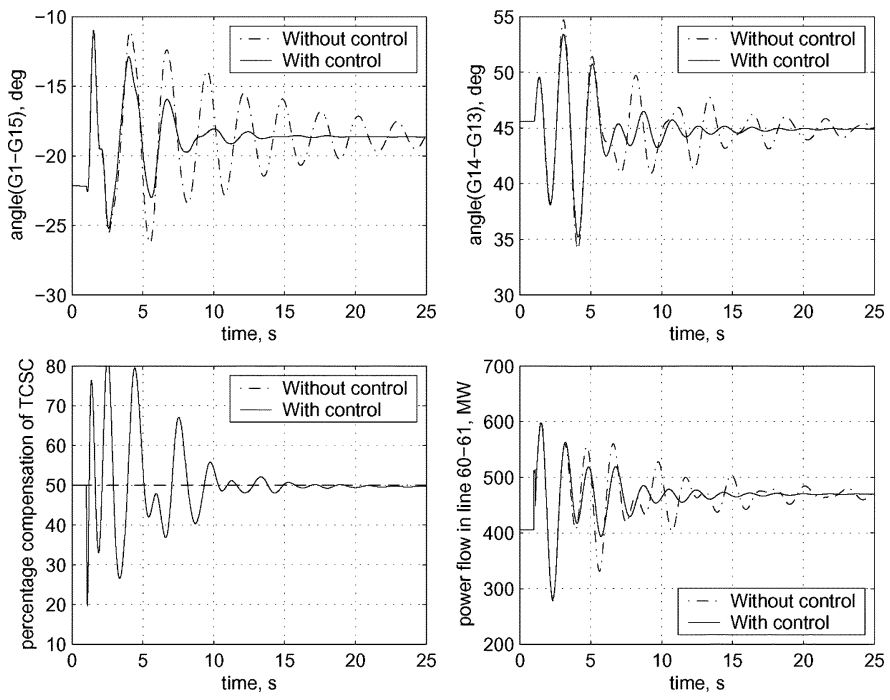

Fig. 9. Test case IIa: Dynamic response of the system.

1) Case IIa: The simulation results for Test Case IIa are shown in Figs. 8 and 9. Contrary to the previous case, the linearized model (model 4) of the power system governing the post-disturbance dynamics and the corresponding controller (controller 4) was intentionally removed from the model bank. As a result, weights corresponding to none of the models attain steady state value of almost 1.0, unlike the previous case (see Fig. 8).

The amount of blending can be adjusted by changing $C_{f}$ and/or $\beta_{\min }$. In this case, the value of $C_{f}$ was chosen to be relatively low (0.0001), as the chances of converging to a single model is less. Also, the magnitude of the artificial cutoff $\beta_{\text {min }}$ was increased to 0.01 to retain even the least probable models. Fig. 9 exhibits the dynamic behavior of the system in response to the same disturbance mentioned in the previous case.

It can be seen that the lightly damped interarea oscillations are settled in 12-15 s. Power flow between buses 60 and 61 also settles within the specified time. The simulation results illustrate that, even though the actual model governing the response
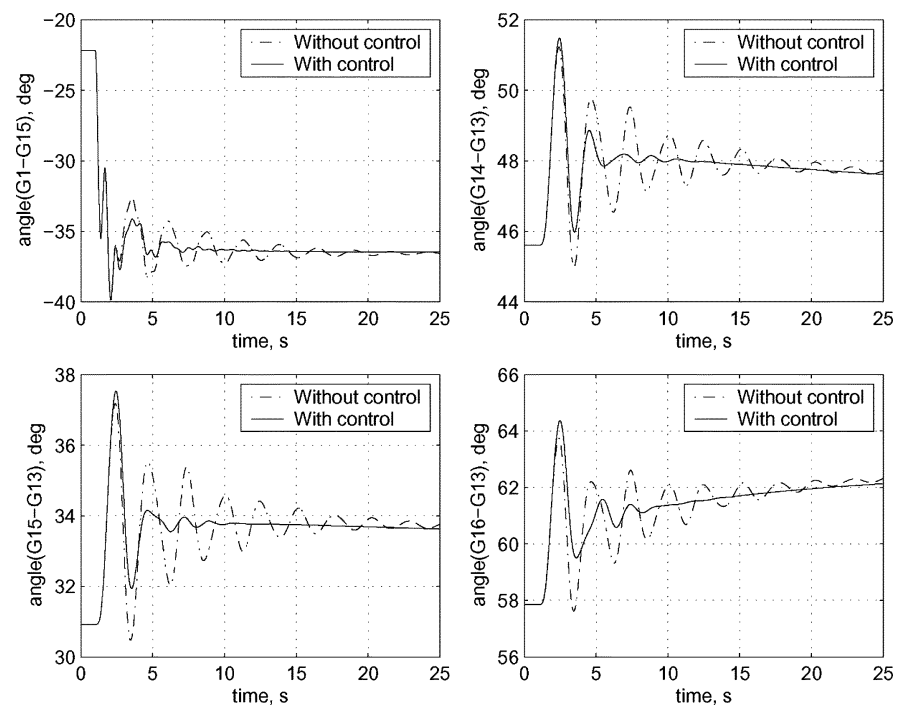

Fig. 10. Test case IIb: Dynamic response of the system.

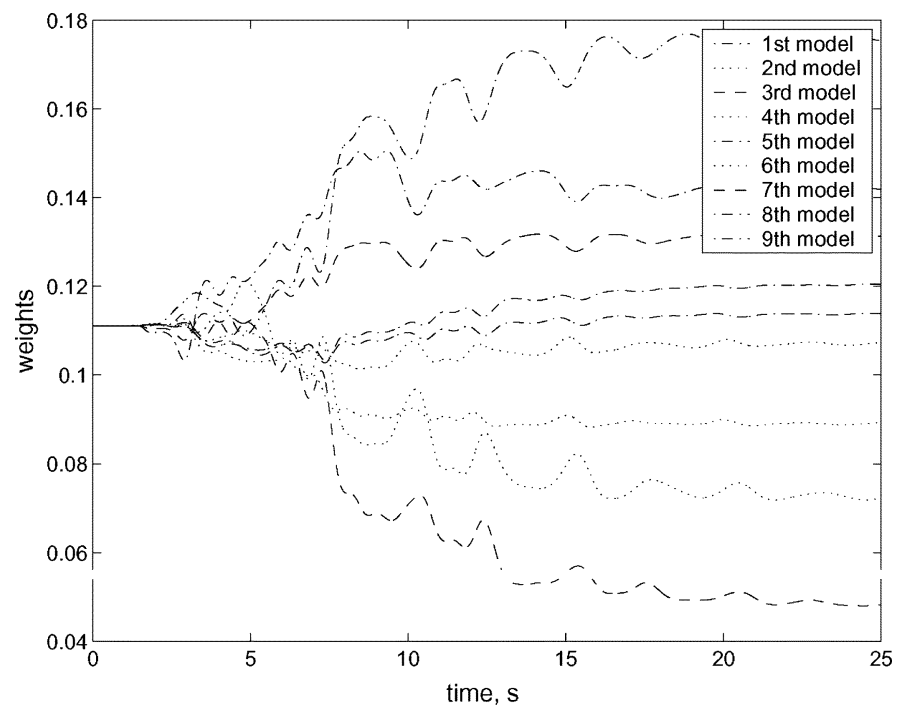

Fig. 11. Test case IIb: Variation of weights.

of the system after the disturbance is absent, the control scheme is able to properly blend the control moves of the remaining controllers and still maintain reasonably similar performance. It can be noted that the compensation of the TCSC varies from $20 \%$ to $80 \%$ in both the cases, as shown in Figs. 7 and 9. The range of variation is relatively large, as a single centralized controller is used to damp all three interarea modes. This is one of the drawbacks of a centralized controller.

2) Case IIb: The simulation results for Test Case IIb are shown in Figs. 10 and 11. In this case, a sudden partial generation loss was considered at generator G1. At $1 \mathrm{~s}$, the generation at generator G1 was reduced to $25 \%$ of its rated capacity. This contingency was not considered while building the linearized models and corresponding controllers. The same values of $C_{f}$ and $\beta_{\min }$ are used, as in Case IIa, to ensure proper blending. The system response is shown in Fig. 10.

The simulation results illustrate that, even though the actual model governing the response of the system after the disturbance is absent, the control scheme is able to properly blend 
the control moves of the remaining controllers and still maintain reasonably similar performance. In fact, no noticeable deterioration can be observed in terms of performance in Fig. 7, when compared with Figs. 9 and 10. This is particularly encouraging, as it makes this probabilistic model-based control scheme a reasonable candidate for application in large practical power systems, where the chances of convergence to a single model are remote. Moreover, during the fault, the dynamics of the system are represented in a more realistic way by a combination of several models rather than by a single model.

\section{CONCLUSION}

This paper has demonstrated the application of a probabilistic approach of a model-based control scheme for adaptive damping of interarea oscillations in power systems using a TCSC. A recursive Bayesian approach is used for computing the current probability of each model representing the actual system response, and the results are used to determine the subsequent control move. The control output of each individual controller is assigned a weight based on the computed probability of each model, and the resulting control action is the probability-weighted average of the control moves of individual controllers. The algorithm is shown to work satisfactorily for the study system under two different test cases, where the model corresponding to the post-disturbance behavior is either present or not present in the model bank. In both cases, the performance of the control scheme was quite satisfactory. This highlights the potential applicability of this control algorithm for large practical power systems where the dynamics are unlikely to be governed by a single model. Under such a situation, the key to the success of the scheme is the rate of convergence of the probabilities, which, in turn, is governed by the proper choice of convergence factor $C_{f}$ and artificial cutoff $\beta_{\min }$.

\section{REFERENCES}

[1] J. Paserba, Analysis and control of power system oscillation, in CIGRE Special Pub. 38.01.07, 1996.

[2] P. Kundur, Power System Stability and Control. New York: McGrawHill, 1994.

[3] N. Hingorani and L. Gyugyi, Understanding FACTS. Piscataway, NJ: IEEE Press, 2000.

[4] O. Malik, G. Hope, and V. Ramanujan, "Real-time model reference adaptive control of synchronous machine excitation," in Proc. IEEE PES Winter Meeting, vol. 178, 1976, pp. 297-304.

[5] N.C. Pahalawaththa, G. S. Hope, and O. P. Malik, "Multivariable selftuning power system stabilizer simulation and implementation studies," IEEE Trans. Energy Convers., vol. 6, no. 2, pp. 310-319, Jun. 1991.

[6] G. Bandyopadhyay and S. Prabhu, "A new approach to adaptive power system stabilizers," Elect. Mach. Power Syst., vol. 14, pp. 111-125, 1988.

[7] J. Hauer, "Robust damping control for large power systems," IEEE Contr. Syst. Mag., vol. 9, no. 1, pp. 12-18, Jan. 1989.

[8] M. Klein, L. Le, G. Rogers, S. Farrokpay, and N. Balu, " $H_{\infty}$ damping controller design in large power system," IEEE Trans. Power Syst., vol. 10, no. 1, pp. 158-166, Feb. 1995.

[9] G. Rogers, Power System Oscillations. Norwell, MA: Kluwer, 2000.

[10] B. Pal, A. Coonick, and B. Cory, "Robust damping of interarea oscillations in power systems with superconducting magnetic energy storage devices," Proc. Inst. Elect. Eng., vol. 146, no. 6, pp. 633-639, Nov. 1999.
[11] N. Martins and L. Lima, "Determination of suitable locations for power system stabilizers and static var compensators for damping electromechanical oscillations in large power systems," IEEE Trans. Power Syst., vol. 5, no. 4, pp. 1455-1469, Nov. 1990.

[12] D. Lainiotis, "Partitioning: a unifying framework for adaptive systems, II: Control," Proc. IEEE, vol. 64, pp. 1182-1198, 1976.

[13] M. Athans, D. Castanon, K.-P. Dunn, C. Greene, W. Lee, N. Sandell, and A. Willsky, "The stochastic control of the F-8C aircraft using a multiplemodel adaptive control MMAC method-part I: Equilibrium flight," IEEE Trans. Autom. Contr., vol. AC-22, no. 5, pp. 768-780, Oct. 1977.

[14] W. He, H. Kaufman, and R. Roy, "Multiple-model adaptive control procedure for blood pressure control," IEEE Trans. Biomed. Eng., vol. BME-33, pp. 10-19, Jan. 1986.

[15] J. Martin, A. Schneider, and N. Smith, "Multiple-model adaptive control of blood pressure using sodium nitroprusside," IEEE Trans. Biomed. Eng., vol. BME-34, pp. 603-611, Aug. 1987.

[16] R. Rao, B. Aufderheide, and B. Bequette, "Experimental studies on multiple-model predictive control for automated regulation of hemodynamic variables," IEEE Trans. Biomed. Eng., vol. 50, no. 3, pp. 277-288, Mar. 2003.

[17] G. J. Anders, Probability Concepts in Electric Power Systems. New York: Wiley, 1990.

[18] D. N. Ewart, "Whys and wherefores of power system blackouts," IEEE Spectr, vol. 15, pp. 36-41, Apr. 1978.

[19] C. Yu, R. Roy, H. Kaufman, and B. Bequette, "Mutiple-model adaptive predictive control of mean arterial pressure and cardiac output," IEEE Trans. Biomed. Eng., vol. 39, no. 8, pp. 765-778, Aug. 1992.

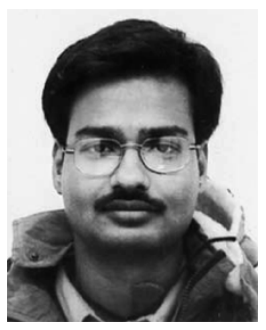

Rajat Majumder (S'04) received the B.E.E. (Hons) degree from Jadavpur University, Calcutta, India, and the M.Sc (Engg) degree from the Indian Institute of Science, Bangalore, India, in 2000 and 2003, respectively. He is currently working toward the Ph.D. at the control and power group, Imperial College London, London, U.K.

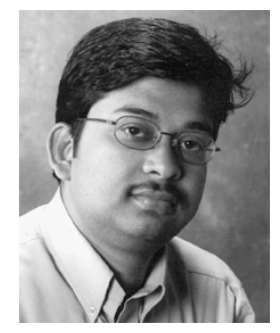

Balarko Chaudhuri (S'02) received the B.E.E. (Hons) degree from Jadavpur University, Calcutta, India, and the M.Tech degree from the Indian Institute of Technology, Kanpur, India, in 2000 and 2002 , respectively. He is currently working toward the Ph.D. degree at the control and power group, Imperial College London, London, U.K.

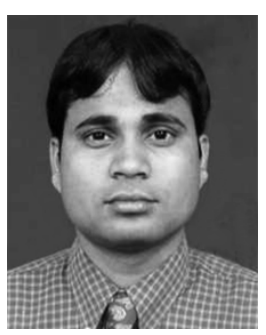

Bikash C. Pal (SM'03) received the B.E.E. (Hons) and the M.E. degrees from Jadavpur University, Calcutta, India, and the Indian Institute of Science, Bangalore, India, in 1990 and 1992, respectively. $\mathrm{He}$ received the $\mathrm{Ph} . \mathrm{D}$. degree from Imperial College London, London, U.K., in 1999.

$\mathrm{He}$ is presently a lecturer in the Department of Electrical and Electronic Engineering, Imperial College London. His research interest is in the area of power system dynamics and FACTS controllers. 